\title{
Potency Combination of Curcumin (Curcuma longa Linn.) and Vitamin E toward Estrogen and Progesterone Profile on Rat (Ratttus norvegicus) Mammary Cancer Model
}

\author{
Herawati, Aulia Firmawati , Dyah Ayu Oktavianie Ardhiana Pratama, and Anna Roosdiana \\ 1,2,3 Faculty of Veterinary Medicine, Brawijaya University, , Brawijaya University, Jl. MT \\ Haryono Malang \\ ${ }^{4}$ Department of Chemistry, Faculty of Science, Brawijaya University, Jl Veteran 65145, \\ Malang, Indonesia*e-mail: auliafirmawatidrh@gmail.com
}

\begin{abstract}
This study aimed to determine the potency of combination of medicinal plants, which is curcumin and vitamin $\mathrm{E}$ as an alternative herbal for breast cancer treatment using DMBA $((7,12-$ Dimethylbenz(a)anthracene) induced rats (Rattus norvegicus) as animal model. The treatments were normal rats as negative control group (NCG), DMBA induced rats as breast cancer suffering rats or positive control group (PCG), and DMBA induced rats treated with combination of curcumin $72 \mathrm{mg}$ per $\mathrm{kg}$ body weight per day and vitamin E $200 \mathrm{IU}$ per head per day for 14 days as treated positive control group 1 (TPCG 1) and DMBA induced rats as breast cancer suffering rats or positive control group (PCG), and DMBA induced rats treated with combination of curcumin $48 \mathrm{mg}$ per $\mathrm{kg}$ body weight per day and vitamin E $300 \mathrm{IU}$ per head per day for 14 days as treated positive control group 2 (TPCG 2). Therapy of the DMBA induced rats was done per orally using combination of curcumin $72 \mathrm{mg}$ per $\mathrm{kg}$ body weight per day and vitamin E 200 IU per head per day for 14 days. After the 14 days, euthanasia was done on the rats and blood samples were collected from each rat for laboratory analysis of estrogen and progesterone concentration using Enzyme Linked Immunosorbent Assay (ELISA) procedure. It was clearly shown that combination of curcumin $72 \mathrm{mg}$ per $\mathrm{kg}$ body weight per day and vitamin E $200 \mathrm{IU}$ per head per day has potential as an anti cancer therapy, as characterized by the decrease of blood estrogen concentration, the increase of blood progesterone concentration, and the disappearance of nodules in the breast tissues of cancer suffering animals.
\end{abstract}

Keywords: Curcumin, Vitamin E, Mammary cancer.

\section{INTRODUCTION}

Cancer is a degenerative disease that is common in humans and pets, especially cats and dogs. Breast cancer has been reported as one of the three types of cancer that most often affects cats, especially female cats [5]. The incidence of cancer, especially breast cancer in pets is often as the result of contraceptive use and ovario hysterectomy. In dogs, the rate of breast cancer incidence is relatively lower than breast cancer incidence in cats. It was reported that the target gene of breast cancer in humans were also found in cats. Several genes that are known as cancer marker genes are p53, BCL-2, ICAM-1 and HER-2. Genes of p53 are known as tumor suppressor genes and BCL-2 are as apoptosis regulator genes. Hence both genes are as cancer control element. A decrease in both genes expression was reported to have correlation with the progression and metastasis of cancer. Whereas over expression of ICAM-1 and HER - 2 can stimulate an increase in cell proliferation triggered motility, invasion and metastasis of cancer cells. Stimulation of cancer can also be seen from the expression of VEGF (Vascular Endothelial 
Growth Factor) as a stimulant of angiogenesis process in cancer formation. It is well known that high plasma estrogen concentration and low plasma progesteron concentration have been measured in many researches and have been used to direct clinical management in patients with breast cancer [7]. High estrogen concentration and increase in lifetime of its exposure were reported as major factors causing breast cancer, as the conditions have a potential in generating free radicals and reactive oxygen species (ROS). Generation of ROS and an altered redox status have long been observed in cancer cells [11]. Recent data showed that ROS could induce DNA synthesis, increased phosphorylation of kinases, and activated transcription factors, e.g., AP-1, NRF1, E2F, NF-kB and CREB of non-genomic pathways which are responsive to both oxidants and estrogen [13].

In relation to the effect of blood progesteron on cancers, [11] reported that high doses of progesterone (P4) caused apoptosis in ovarian and endometrial cancer cells with $\mathrm{P} 4$. This implicated that P4 might have potential as a protective factor for ovarian and endometrial cancers, which meant that higher blood concentration of progesteron should decrease the potential of cancer incident, although the mechanism of the protection is little known. Several efforts to minimize cancer in animals were commonly done with surgical therapy for elimination of cancer nodules [5]. Treatment or prevention of cancer using herbals is rarely used and has not been reported. On the other hand, the uses of herbal medicine for health treatment become more popular because the use of herbal medicines has been known not to have side effects as compared to the use of synthetic medicines. Several studies reported that herbal medicines have potential as carcinoma or cancer therapy [14]. One of the traditional herbal medicines that can be used to treat cancer is curcumin, which is the active ingredient commonly extracted from Curcuma longa. Curcumin is a natural yellow pigment in turmeric. It is a polyphenolic compound, which has biological activity as an antioxidant, anti-inflammatory, chemopreventive and chemotherapeutic [1] [9] [14]. Curcumin was also known to have activity as anti cancer, anti mutagenic, anti coagulant, anti fertility, anti diabetic, anti bacterial, anti fungal, anti protozoa, anti viral as well as anti fibrosis [18].

Anti cancer activity of curcumin was reported to be associated with its activities as an antioxidant that has been known to function as scavenger and catcher of free radical or ROS as promotor of many aspects of tumour development and prolification [15]. ROS concentration has been detected to increase sharply in almost all cancers incident [16]. Curcumin is a broadspectrum anti-cancer agent and it was declared that curcumin is as a potential material for the prevention and treatment of cancer, which can suppress tumor initiation, promotion and metastasis [8].

This research aimed to determine the potency of curcumin and vitamin E combination as an alternative antioxidant used in treatment of breast cancer using DMBA $((7,12$ dimethylbenz(a) anthracene ) induced rats (Rattus norvegicus) as cancer suffering animal model as also used in the research of [3] [12] [17], based on blood estrogen and progesterone concentration. It is expected that the results of this study can be used as a basis for the development of alternative herbal therapy for cancer treatment, especially in pets.

\section{MATERIALS AND METHODS}

Twenty one female rats (Rattus norvegicus) Sprague Dawley strain, 10-12 weeks of age and 180-250 $\mathrm{g}$ of live weight were allotted into completely randomized design of three 
treatments and seven replications. The treatments were normal rats as negative control group (NCG), DMBA induced rats as breast cancer suffering rats or positive control group (PCG), and DMBA induced rats daily treated with combination of curcumin and vitamin $\mathrm{E}$ as treated positive control group (TPCG). Each treatment was applied to seven rats as replication.

DMBA induction was done subcutaneously intra mammary injection on both left and right sides flank using multiple low dosages of $10 \mathrm{mg} / \mathrm{kg}$ body weight for 10 times injection every two days. Therapy of the DMBA induced rats was done per orally using combination of curcumin $72 \mathrm{mg}$ per kg body weight per day and vitamin E $200 \mathrm{IU}$ per head per day for 14 days (Pari, 2008). After the 14 days, euthanasia was done on the rats and blood samples were collected from each rat for laboratory analysis of estrogen and progesterone concentration using Enzyme Linked Immunosorbent Assay (ELISA) procedure. Data collected were subjected to analysis of variance according to completely randomized design.

\section{RESULTS}

Blood concentration of estrogen and progesterone of rats on each treatment was presented on Figure 1.Data on Figure 1 show that blood estrogen concentration of DMBA induced rats $(\mathrm{PCG})$ was significantly higher $(\mathrm{P}<0.01)$ than those in the normal rats $(\mathrm{NCG})$. So that, it was clearly shown that DMBA induction increased significantly blood estrogen concentration. DMBA induction led to the increase of the estrogen receptors (ER) in the breast epithelial cells. Physiologically, estrogen is one of reproductive hormones that play a role in the proliferation of breast cells. In the process of breast cell proliferation, estrogen binds to the estrogen receptors (ER) in the epithelial cells of breast, ovarian and endometrial to assist the process of cell proliferation, cell differentiation and contributes to the physiological function of reproduction. If the estrogen receptors (ER) do not bind to estrogen due to over expression, and then most of the estrogen receptors (ER) are located in the cytosol. Over expression of these receptors trigger the displacement of estrogen receptors (ER) and move from the cytosol to the nucleus, and then bind to DNA. DNA- estrogen receptors (ER) complexes formed then regulate protein synthesis that lead to the changes in cell function. Moreover, in the case of breast cancer that occurs in mice models, over expression of estrogen receptors (ER) will stimulate gene expression of a number of cancers, such as Human Epidermal Growth Receptors 2 (HER-2). HER-2 encodes a tran membrane glycoprotein with tyrosine kinase activity, which plays a role in the regulation of growth cell neoplasia. Some evidence for cross-talk between ER and p53 pathways at several levels suggests that the impact of TP53 mutation may be affected by the presence of ER. First, ER is a p53 target and conversely estrogen treatment induces p53 expression [4].

On the other hand, data on Figure 1 show that blood concentration of progesterone of DMBA induced rats $(\mathrm{PCG})$ was significantly lower $(\mathrm{P}<0.05)$ than those in the normal rats (NCG) and DMBA induced rats treated using combination of curcumin and vitamin E (TPCG. The data gave further justification on the curative effect of therapy using combination of curcumin and vitamin $\mathrm{E}$ on cancer. Both curcumin compound and vitamin $\mathrm{E}$ were very well known to have potential function as an antioxidant. Hence, the antioxidant inhibit the formation of free radicals by suppressing the activity of cytochromep450 [10]. The chemopreventive agent of curcumin has been known as antioxidant and tumor cell radiosensitizing properties. Curcumin was known to have function in inhibiting the proliferation of breast cancer cells (BT20, SKBR3, 
MCF-7, T47D and ZR75-I types) in vitro [2]. The anti-proliferation effect is highly dependent on estrogen receptors (ER) positive. Inhibition of activation of the estrogen receptors (ER) would result in inhibition of the activity of several transcription factors through induction of RNA polymerase that can inhibit cancer cell proliferation.

\section{DISCUSSION}

Blood estrogen concentration of DMBA induced rats treated with combination of curcumin and vitamin E (TPCG) was significantly lower than those in the DMBA induced rats (PCG) but closely similar to those in normal rats (NCG). These showed clearly that therapy using combination of curcumin and vitamin $\mathrm{E}$ on DMBA induced rats or breast cancer suffering rats (PCG) decreased significantly blood estrogen concentration as in the normal rats. This meant that therapy using combination of curcumin and vitamin $\mathrm{E}$ has potential curative function on breast cancer suffering animals.

Physical conditions of breast tissues of DMBA induced rats (PCG) as compared to those in the DMBA induced rats treated using combination of curcumin and vitamin E (TPCG) was shown on Figure 2. The figure shows clearly that there are some nodules on the breast tissues of DMBA induced rats group (PCG). However, the nodules were not found on the breast tissues of the DMBA induced rats treated using combination of curcumin and vitamin E (TPCG). The present of nodules on the breast tissues is well known to be one of indicator of breast cancer incident. Mammary DMBA induction on rats caused neoplasm in mammary cell. Neoplasm causes in changes of mammary tissues. The occurrence was found in $75 \%$ of rats after the DMBA induction. The present of nodules and their development (increase in size and number) caused mammary tissues harder, which is very well known as one of indicator of cancer incident on breast tissues.

\section{REFFERENCES}

1) Aftab N, Vieira A. Antioxidant activities of curcumin and combinations of this curcuminoid with other phytochemicals. Phytother. Res 2010, 24, 500-502. Epub ahead of print doi: 10.1002/ptr.2960.

2) Aggarwal A, Prabakaran SA. Oxidative Stress and Antioxidants in Male Infertility: a Difficult Balance. Iran J Reprod Med 2005, 3, 1-8.

3) Chen YK, Lin LM. DMBA-induced hamster buccal pouch carcinoma and VX2- induced rabbit cancer as a model for human oral carcinogenesis. Expert Rev Anticancer Ther 2010, 9, 1485-1496. 